\title{
Three-dimensional simulations of gravitationally confined detonations compared to observations of SN 1991T
}

\author{
Ivo R. Seitenzahl ${ }^{1,2,3}$, Markus Kromer ${ }^{4}$, Sebastian T. Ohlmann ${ }^{5}$, Franco Ciaraldi-Schoolmann ${ }^{1}$, Kai Marquardt ${ }^{5,6}$, \\ Michael Fink $^{6}$, Wolfgang Hillebrandt ${ }^{1}$, Rüdiger Pakmor ${ }^{5}$, Friedrich K. Röpke ${ }^{5,7}$, Ashley J. Ruiter ${ }^{2,3}$, \\ Stuart A. $\operatorname{Sim}^{8}$, and Stefan Taubenberger ${ }^{1}$
}

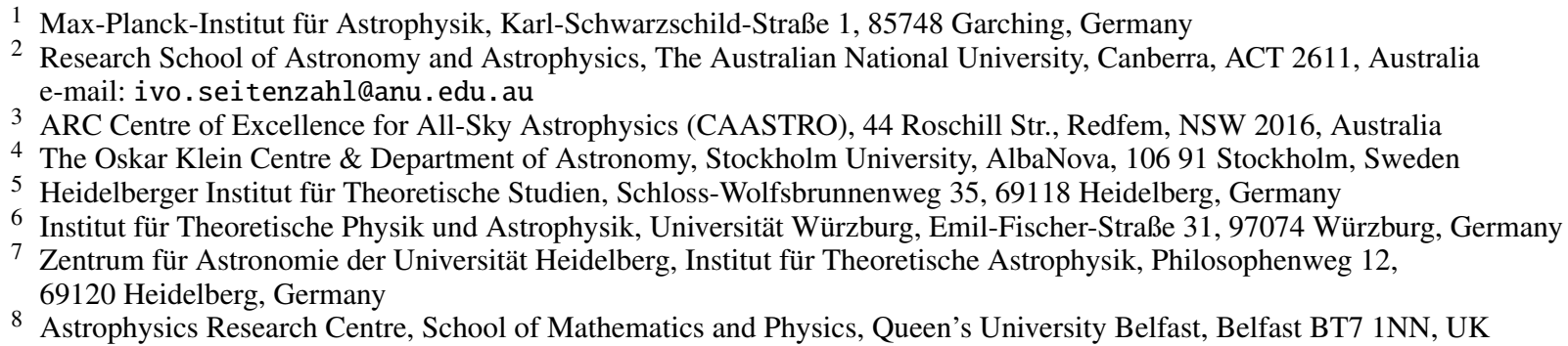

Received 26 August 2015 / Accepted 31 May 2016

\begin{abstract}
The gravitationally confined detonation (GCD) model has been proposed as a possible explosion mechanism for Type Ia supernovae in the single-degenerate evolution channel. It starts with ignition of a deflagration in a single off-centre bubble in a near-Chandrasekharmass white dwarf. Driven by buoyancy, the deflagration flame rises in a narrow cone towards the surface. For the most part, the main component of the flow of the expanding ashes remains radial, but upon reaching the outer, low-pressure layers of the white dwarf, an additional lateral component develops. This causes the deflagration ashes to converge again at the opposite side, where the compression heats fuel and a detonation may be launched. We first performed five three-dimensional hydrodynamic simulations of the deflagration phase in $1.4 M_{\odot}$ carbon/oxygen white dwarfs at intermediate-resolution $\left(256^{3}\right.$ computational zones). We confirm that the closer the initial deflagration is ignited to the centre, the slower the buoyant rise and the longer the deflagration ashes takes to break out and close in on the opposite pole to collide. To test the GCD explosion model, we then performed a high-resolution $\left(512^{3}\right.$ computational zones) simulation for a model with an ignition spot offset near the upper limit of what is still justifiable, $200 \mathrm{~km}$. This high-resolution simulation met our deliberately optimistic detonation criteria, and we initiated a detonation. The detonation burned through the white dwarf and led to its complete disruption. For this model, we determined detailed nucleosynthetic yields by post-processing $10^{6}$ tracer particles with a 384 nuclide reaction network, and we present multi-band light curves and time-dependent optical spectra. We find that our synthetic observables show a prominent viewing-angle sensitivity in ultraviolet and blue wavelength bands, which contradicts observed SNe Ia. The strong dependence on the viewing angle is caused by the asymmetric distribution of the deflagration ashes in the outer ejecta layers. Finally, we compared our model to SN 1991T. The overall flux level of the model is slightly too low, and the model predicts pre-maximum light spectral features due to $\mathrm{Ca}$, S, and $\mathrm{Si}$ that are too strong. Furthermore, the model chemical abundance stratification qualitatively disagrees with recent abundance tomography results in two key areas: our model lacks low-velocity stable Fe and instead has copious amounts of high-velocity ${ }^{56} \mathrm{Ni}$ and stable Fe. We therefore do not find good agreement of the model with SN 1991T.
\end{abstract}

Key words. hydrodynamics - radiative transfer - methods: numerical - nuclear reactions, nucleosynthesis, abundances supernovae: general - supernovae: individual: SN $1991 \mathrm{~T}$

\section{Introduction}

It is widely accepted that Type Ia supernovae (SNe Ia) are thermonuclear explosions of white dwarf stars. Since isolated white dwarfs are stable objects, the progenitor star must be interacting with a companion such that critical conditions necessary for explosion can be achieved. These critical conditions vary depending on the explosion mechanism, of which several have been proposed.

The manner in which the exploding, probably carbonoxygen (CO), white dwarf (WD) accretes matter in the first place and the nature of the companion have been topics of debate for decades (Whelan \& Iben 1973; Nomoto 1982; Iben \& Tutukov 1984; Webbink 1984; Yungelson et al. 1995; Tutukov \& Yungelson 1996; Yungelson \& Livio 2000; Han \& Podsiadlowski 2004; Ruiter et al. 2009, 2013; Mennekens et al. 2010; Toonen et al. 2012; Hillebrandt et al. 2013).

Different progenitor evolution scenarios exist, and for some scenarios yet again different explosion models have been proposed. For the single-degenerate scenario, for example, for pure deflagrations, deflagration-to-detonation transitions, pulsational reverse detonations, and gravitationally confined detonation (GCD) models have been suggested. Here we focus on the GCD model, first discussed by Plewa et al. (2004). 
The GCD model starts with the ignition of a deflagration in a single off-centre bubble in a near-Chandrasekhar-mass (near$M_{\mathrm{Ch}}$ ) white dwarf (WD). Driven by buoyancy, the burning products of the deflagration quickly rise towards the stellar surface, where, in addition to the dominant radial expansion, they develop a lateral velocity component and converge at the opposite side. There, the flow compresses and heats still unburned fuel, and a detonation may be launched. To successfully trigger the detonation, flow convergence in rather dense material is required. Therefore, a weak deflagration and only a modest expansion of the WD is expected to favour the scenario (see Sect. 2.2). Consequently, the ensuing detonation burns a large amount of fuel at high densities, mostly to ${ }^{56} \mathrm{Ni}$, thus producing bright $\mathrm{SNe}$.

Supernova 1991T (Filippenko et al. 1992; Phillips et al. 1992; Schmidt et al. 1994; Lira et al. 1998) is the prototypical event of a spectroscopically peculiar class of energetic and luminous SNe Ia. SN $1991 \mathrm{~T}$ is the best characterised exemplar of a sub-class ( $\mathrm{SNe} 91 \mathrm{~T}$ ) of $\mathrm{SNe}$ Ia that make up a few percent of all observed SNe Ia (Li et al. 2011; Silverman et al. 2012; Blondin et al. 2012). In contrast to normal SNe Ia, SNe $91 \mathrm{~T}$ are known to occur preferentially in late-type galaxies (Hamuy et al. 2000; Howell 2001), indicating an origin in young stellar populations. SNe 91T further clearly distinguish themselves from normal SNe Ia by their peculiar pre-maximum light spectra. In particular, the characteristic Si II $\lambda \lambda 5972,6355$ features prominent in normal SNe Ia (e.g. Branch et al. 1993) are essentially absent before maximum light; the same holds for other features of intermediate-mass elements, such as Ca II or S II. Instead, early spectra show unusual Fe III features (Filippenko et al. 1992; Ruiz-Lapuente et al. 1992; Jeffery et al. 1992), requiring high ionisation and $\mathrm{Fe}$ abundance at high velocity. Moreover, SNe $91 \mathrm{~T}$ events are more luminous by about $0.2-0.3 \mathrm{mag}$ than the width-luminosity relation predicts for normal $\mathrm{SNe}$ Ia (Blondin et al. 2012).

At first sight, it is tempting to liken the GCD models with SNe $91 \mathrm{~T}$ (e.g. Fisher \& Jumper 2015). The deflagration ashes present at high velocities in the outer layers of GCD models result in a chemically mixed composition that is highly enriched with Fe-group isotopes at high velocities, which could explain key results obtained from interpreting observations of SN 1991T (e.g. Mazzali et al. 1995; Fisher et al. 1999; but see Sasdelli et al. 2014). Furthermore, the fact that only a few per cent of SNe Ia are classified as 1991T-like (Li et al. 2011; Silverman et al. 2012; Blondin et al. 2012) would be naturally explained by the relative scarcity of the single-degenerate scenario compared to the violent-merger or double-detonation scenarios (e.g. Ruiter et al. 2009, 2011, 2013; Fisher \& Jumper 2015). The identification of GCD explosions with SNe 91T is challenged from observations of the supernova remnant SNR 0509-67.5 in the LMC. First, based on light echo spectra, Rest et al. (2008) demonstrated clearly that this SN was a 1991T-like explosion. Second, Schaefer \& Pagnotta (2012) ruled out the existence of a companion star in this SNR to very deep limits and thereby argued for a double-degenerate progenitor, which would exclude the canonical single-degenerate formation channel as a path to the GCD model. We note that García-Senz et al. (2016) have recently questioned the viability of the mechanism altogether, arguing that the Coriolis force substantially breaks the symmetry and thereby disfavours the emergence of a detonation.

Historically, both delayed-detonations in near- $M_{\mathrm{Ch}}$ WDs (Mazzali et al. 1995) and sub-Chandrasekhar-mass (sub- $M_{\mathrm{Ch}}$ ) double detonations (DDs; e.g. Liu et al. 1997) have been suggested as possible explosion models for SN 1991T. However, detailed comparisons of SN $1991 \mathrm{~T}$ and synthetic spectra and light curves of bright sub- $M_{\mathrm{Ch}}$ DD models (Kromer et al. 2010; Woosley \& Kasen 2011) and bright near- $M_{\mathrm{Ch}}$ delayeddetonation models (Sim et al. 2013) found substantial disagreement between the model predictions and the observations. Similarly, pure detonations of ONe WDs (Marquardt et al. $2015)$, which can produce SNe Ia with ${ }^{56} \mathrm{Ni}$ masses around $1 M_{\odot}$, are inadequate models for $\mathrm{SNe} 91 \mathrm{~T}$ (for example, the strong Si II and $\mathrm{Ca}$ II absorption features present in the models before maximum light are almost absent in SNe 91T).

Although successful explosions of near- $M_{\mathrm{Ch}}$ WDs in the GCD-framework have been obtained in several hydrodynamical explosion simulations (Plewa 2007; Meakin et al. 2009; Jordan et al. 2012a), only Meakin et al. (2009) have presented detailed isotopic yields for their 2D models. Kasen \& Plewa (2007) presented broadband optical and near-infrared light curves, spectral time series, and spectropolarisation for their Y12 model and were generally successful in reproducing the basic properties of observed SNe Ia.

In this work, we present (multi-band) light curves and timedependent optical spectra for an explosion model representative of the class of GCD models, for which we employed a detailed treatment of the nucleosynthesis for the detonation as well as for the deflagration. In Sect. 2 we briefly discuss our series of intermediate-resolution, single-bubble, off-centre deflagration simulations and present the evolution of a high-resolution model that met our very optimistic detonation criteria. In Sect. 4 we present spectra and light curves for this model and compare them with observations. In Sect. 5 we summarise and conclude that these synthetic observables do not resemble any known subclass of SNe Ia.

\section{Hydrodynamic explosion simulations}

According to theory, a detonation may spontaneously be initiated if a sufficiently shallow induction-time gradient can be set up to facilitate the formation of a shock in the shock-wave amplification through coherent energy release (SWACER) mechanism (see e.g. Seitenzahl et al. 2009b). In the GCD model, suitable induction-time gradients may be obtained in the collision region near the stagnation point of the surface flow, directly opposite to the point of breakout of the rising deflagration bubble (Seitenzahl et al. 2009a). Since the spatial scales relevant to the initiation of a detonation cannot be resolved in full-star multi-dimensional explosion simulations (see the discussion in Seitenzahl et al. 2009a), it is common practice (e.g. Jordan et al. 2008, 2012a; Guillochon et al. 2010; Pakmor et al. 2011, $2012 \mathrm{~b})$ to pick a certain critical density $\rho_{\text {crit }}$ and temperature $T_{\text {crit }}$ that a cell composed of nuclear fuel must exceed for a detonation to be initiated. However, these critical values are no more than informed guesses based on separate high-resolution onedimensional detonation initiation calculations (e.g. Niemeyer \& Hillebrandt 1997; Seitenzahl et al. 2009b).

\subsection{Computational method}

We performed three-dimensional full-star simulations with the hydrodynamic supernova explosion code LEAFS. The whole exploding WD and the deflagration flame are captured separately on two different nested co-moving grids (Röpke 2005; Röpke et al. 2006). Since the flame is very thin compared to the radius of a near- $M_{\mathrm{Ch}} \mathrm{WD}$, the former is treated as a sharp discontinuity with the help of the level set technique (Osher \& Sethian 1988; Reinecke et al. 1999). The effective burning speed of the 
deflagration flame is composed of a laminar and a turbulent contribution (Pocheau 1994). For the former we used tabulated values from Timmes \& Woosley (1992), while the turbulent flame speed was determined from a subgrid-scale turbulence model of Schmidt et al. (2006a,b). The energy release behind the deflagration level set is determined as a function of fuel density by interpolation in a table that is consistent with the energy release of a detailed nuclear reaction network; for details see Appendix A of Fink et al. (2014). The detonation was also modelled with a (separate) level set. For the detonation speed and energy release we used the tables of Fink et al. (2010), which, just as the deflagration tables, take the effects of incomplete burning at low densities into account. The reactive Euler equations are numerically solved with a finite-volume method (Fryxell et al. 1989) based on the piecewise-parabolic method (PPM) of Colella \& Woodward (1984). For the nucleosynthesis, we post-processed one million tracer particles with a nuclear reaction network using the technique described in Travaglio et al. (2004) and Seitenzahl et al. (2010). The simulation code is essentially the same as described in Seitenzahl et al. (2013b), a key difference is, however, that the deflagration-to-detonation transition module was de-activated and the initiation of detonations is handled in a different way.

Here, we initialised the detonation around the grid cell in which both critical values $\rho_{\text {crit }}$ and $T_{\text {crit }}$ were (simultaneously) reached or exceeded first. Furthermore, to avoid artificial numerical triggering of the detonation, we required that the deflagration level set was at least one grid cell away and that the grid cell was composed of at least 90 per cent of unburned ${ }^{16} \mathrm{O}$ and ${ }^{12} \mathrm{C}$.

Our initial stellar model was an isothermal near- $M_{\mathrm{Ch}} \mathrm{WD}$ with a central density of $2.9 \times 10^{9} \mathrm{~g} \mathrm{~cm}^{-3}$ and a temperature of $5 \times 10^{5} \mathrm{~K}$. For the hydrodynamical simulations, the stellar material was assumed to be composed of carbon and oxygen where we approximated the effect of the assumed solar metallicity of the zero-age main-sequence progenitor on the stellar structure by initializing the electron fraction to $Y_{\mathrm{e}}=0.49886$ (see e.g. Seitenzahl et al. 2011).

\subsection{Moderate-resolution test cases}

We ignited the deflagration in a single spherical off-centre region with a bubble radius of $10^{6} \mathrm{~cm}$ and carried out simulations of five models at a moderate resolution of $256^{3}$ grid cells. The models varied only in the distance $d_{\mathrm{k}}$ of the centre of the ignition kernel to the centre of the white dwarf, and we tried $d_{\mathrm{k}}=12,37,64,125$, and $200 \mathrm{~km}$. All ignitions occurred at a density greater than $2.3 \times 10^{9} \mathrm{~g} \mathrm{~cm}^{-3}$.

The closer the initial deflagration is ignited to the centre, the slower the buoyant rise and the longer the deflagration ashes take to break out and close in on the opposite pole to collide. Since the white dwarf in the meantime continues to expand, obtaining high temperatures in the collision region at densities higher than $\rho_{\text {crit }}$ becomes less probable. We thus find, as in Röpke et al. (2007), that larger distances of the ignition kernel from the centre of the white dwarf lead to more suitable conditions for a detonation at the closing point of the deflagration ashes. The reason is that the deflagration evolves faster, burns less mass, and releases less energy, which consequently leads to less pronounced expansion of the WD, so that when the erupted deflagration ashes converge, sufficiently high temperatures are more readily obtained at densities where detonations can occur (Townsley et al. 2007; Röpke et al. 2007; Aspden et al. 2011); see Malone et al. (2014) for an explanation of how background turbulence of the convective flow moderates this trend for very small offsets.

\subsection{High-resolution simulation of model GCD200}

Our main motivation for this work is not to elucidate whether a detonation can be triggered in the GCD framework, but rather what the predicted observables of the model look like if a detonation is triggered. We therefore chose a large offset for the ignition of the deflagration, $200 \mathrm{~km}$, which is more favourable to attaining detonation conditions than smaller offsets are. While $200 \mathrm{~km}$ is still commensurate with the analytical calculations of Garcia-Senz \& Woosley (1995) and Woosley et al. (2004), the numerical simulations of Kuhlen et al. (2006) and Nonaka et al. (2012) favour much smaller offset distances. It is therefore questionable whether an offset of $200 \mathrm{~km}$ for the ignition point is attainable at all.

Previous 3D hydrodynamics simulations with the LEAFS code (see Table 4 of Röpke et al. 2007) failed to obtain the more conservative detonation conditions $\rho_{\text {crit }}=10^{7} \mathrm{~g} \mathrm{~cm}^{-3}$ and $T_{\text {crit }}=$ $1.9 \times 10^{9} \mathrm{~K}$, even for offset distances of the initial deflagration bubble as large as $200 \mathrm{~km}$. Since we focus here on the model implications if a detonation occurs, we chose lower, less restrictive values for this work: $\rho_{\text {crit }}=10^{6} \mathrm{~g} \mathrm{~cm}^{-3}$ and $T_{\text {crit }}=1.0 \times 10^{9} \mathrm{~K}$. We also required the fuel content of the cell to be greater than 90 per cent, that is, $X_{\text {fuel }}>0.9$. We note that this choice of critical detonation conditions is very optimistic, pushing the boundaries of the parameter space where detonations are expected to form through the gradient mechanism (Seitenzahl et al. 2009b).

We simulated a single-bubble ignition model with a bubble radius of $10^{6} \mathrm{~cm}$ and $d_{\mathrm{k}}=200 \mathrm{~km}$, with an increased resolution of $512^{3}$ grid cells (hereafter referred to as GCD200). This highresolution model evolves to meet our critical detonation conditions (outlined above) at $t=2.37 \mathrm{~s}$, and we initialised a single detonation around the grid cell where the constraints are first satisfied. The detonation was initiated by adding a second level set at this instant with positive values in a sphere of a radius of three cells $(330 \mathrm{~km})$ and converting this material instantaneously to iron group elements.

In Fig. 1 we show a two-dimensional slice of the temperature for two instants of time (2.20 s and 2.37 s). Most material at densities above $10^{6} \mathrm{~g} \mathrm{~cm}^{-3}$ is unburned and the WD core (densities above $10^{7} \mathrm{~g} \mathrm{~cm}^{-3}$ ) appears spherical and hardly distorted by the deflagration. In the second snapshot, which corresponds to the time when we initiated the detonation, a hotspot is forming in the focus region in unburned material where the ashes of the deflagration converge. The central density of the white dwarf at this time $(2.37 \mathrm{~s})$ is $6.63 \times 10^{7} \mathrm{~g} \mathrm{~cm}^{-3}$. The density and temperature in the grid cell around which the detonation is initialised are $\rho_{\text {fuel }}=1.09 \times 10^{6} \mathrm{~g} \mathrm{~cm}^{-3}$ and $T_{\text {fuel }}=1.02 \times 10^{9} \mathrm{~K}$. These thermodynamic conditions only satisfy the critical detonation criteria for this work, but would not have satisfied the more restrictive critical conditions of Röpke et al. (2007). Although our model was far from the more canonical and arguably more realistic detonation criteria $\rho_{\text {crit }} \approx 1.0 \times 10^{7} \mathrm{~g} \mathrm{~cm}^{-3}$ and $T_{\text {crit }} \approx 2.0 \times 10^{9} \mathrm{~K}$ (Seitenzahl et al. 2009b), we cannot conclude about the likelihood of the formation of a detonation in GCD models in general. Our particular model represents only one specific realisation, albeit with a choice of offset of the initial deflagration bubble that is already most favourable for a detonation. For different setups or ignition parameters, for example different choices for the central density or composition, the situation may be different. 

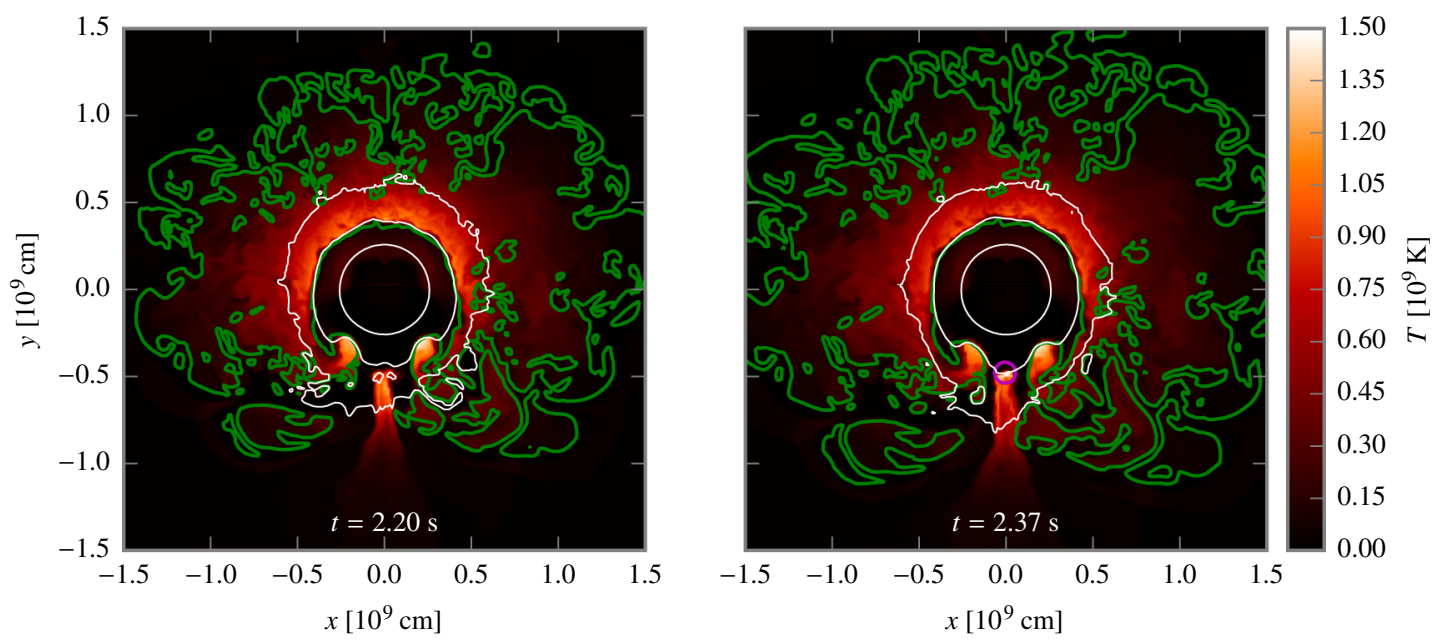

Fig. 1. 2D slice through the midplane of the simulation volume along the $x$-and $z$-axes for model GCD200 at $t=2.20 \mathrm{~s}($ left panel $)$ and at $t=2.37 \mathrm{~s}$ (right panel) with colour-coded temperature. The green contour line displays the position of the deflagration level set; any material enclosed by a green line has been burned in the deflagration. The white contour lines show the density at $10^{7} \mathrm{~g} \mathrm{~cm}^{-3}, 10^{6} \mathrm{~g} \mathrm{~cm}^{-3}$, and $10^{5} \mathrm{~g} \mathrm{~cm}^{-3}$ (from the centre outwards). The left panel shows the temperature slightly before the detonation is initialised, the right panel the temperature at the time of the initialisation of the detonation. The detonation spot is shown by a magenta circle (right panel).

The progression of the explosion after the onset of the detonation is visualised in Fig. 2. The initialisation of the detonation, which occurs at $t \approx 2.37 \mathrm{~s}$, is shown in the upper left panel of Fig. 2, where the detonation region is encircled. The detonation front propagates towards the central region of the white dwarf, which is predominantly composed of unburned ${ }^{16} \mathrm{O}$ and ${ }^{12} \mathrm{C}$. Since the central density is still rather high, most of the stellar material overrun by the detonation is burned to iron group elements (IGEs), in particular to ${ }^{56} \mathrm{Ni}$. After burning ceases, the detonation ashes are surrounded by the deflagration ashes for most lines of sight, except for a small opening angle of reduced contamination around the detonation initiation site; see Fig. 2f.

In Fig. 3 we show the temporal evolution of the total $\left(E_{\mathrm{tot}}\right)$, nuclear $\left(E_{\text {nuc }}\right)$, kinetic $\left(E_{\text {kin }}\right)$, internal $\left(E_{\text {int }}\right)$, and gravitational energy $\left(E_{\text {grav }}\right)$ as functions of time. Energy conservation demands $E_{\mathrm{tot}}=E_{\mathrm{kin}}+E_{\mathrm{int}}+E_{\text {egrav }}-E_{\text {nuc }}$. We note that the sub-grid scale energy, which is of the order of $10^{47}$ to $10^{48} \mathrm{erg}$ in turbulent deflagrations, is neglected here (see Fig. 1 in Schmidt et al. 2006b). Figure 3a shows model GCD200, while Fig. 3b shows the energies for the corresponding pure deflagration model, where no detonation is initialised. For the GCD simulation, the nuclear energy release is sufficient to unbind the entire white dwarf $\left(E_{\text {grav }}\right.$ asymptotically approaches zero in the late explosion phase). If no detonation occurs, we obtain a supernova explosion that fails to fully unbind the WD and leaves behind a compact remnant after the deflagration flame extinguishes (Jordan et al. 2012b; Fink et al. 2014). Events like this have been shown to reproduce the observable characteristics of SN 2002cx-like SNe (Kromer et al. 2013).

\section{Nucleosynthesis}

Our postprocessing calculations are based on the 384 isotopes nuclear reaction network YANN (Pakmor et al. 2012a) that is run on the thermodynamic trajectories recorded by the tracer particles. The network employs the reaction rates from the JINA database (Cyburt et al. 2010) as of January 27, 2014. As outlined in Kromer et al. (2013), we implemented the effects of solar metallicity of the progenitor by initializing the tracer particle composition to $50 \%{ }^{16} \mathrm{O}, 48.29 \%^{12} \mathrm{C}$, and the remaining
$1.71 \%$ of the mass to the solar composition from Asplund et al. (2009) for elements heavier than He, with the exception of solar $\mathrm{C}, \mathrm{N}, \mathrm{O}$, which we converted into ${ }^{22} \mathrm{Ne}$ to account for the effects of core He-burning.

The masses of stable isotopes after decaying all radioactive nuclides are given in Table 1. Radioactive species $100 \mathrm{~s}$ after ignition are listed in Table 2 . Burning in both deflagration and detonation yields $0.742 M_{\odot}$ of ${ }^{56} \mathrm{Ni}$ and $0.030 M_{\odot}$ and $0.037 M_{\odot}$ of the stable iron group isotopes ${ }^{54} \mathrm{Fe}$ and ${ }^{58} \mathrm{Ni}$, respectively. The former is in large parts synthesised in the detonation phase, while the latter two also formed copiously in the deflagration near the centre where high densities favour neutronisation by electron capture reactions. However, the stable iron group isotopes synthesised in the deflagration do not remain at low velocity. They are carried towards the surface of the WD in the rising deflagration plumes and end up at the highest velocities, see Figs. 4 and 5.

Noteworthy, the GCD200 model has a sub-solar Mn-toFe production ratio of $[\mathrm{Mn} / \mathrm{Fe}]=-0.13$. This sub-solar ratio is a reflection of the fact that when the detonation is initiated, the central density of the WD has already fallen below the separation density between normal and $\alpha$-rich freeze-out, $\rho<2 \times 10^{8} \mathrm{~g} \mathrm{~cm}^{-3}$ (Thielemann et al. 1986; Bravo \& MartínezPinedo 2012), where proton captures during the $\alpha$-rich freezeout cause a lower Mn-to-Fe production ratio (e.g. Jordan et al. 2003; Seitenzahl et al. 2013a). Regardless of occurrence rate and although it originates from exploding near- $M_{\mathrm{Ch}}$ WDs, our GCD model of near- $M_{\mathrm{Ch}} \mathrm{SN}$ Ia explosions therefore cannot explain the solar Mn-to-Fe ratio (see Seitenzahl et al. 2013a).

Using a smoothed-particle-hydrodynamics-like algorithm that approximately conserves the integrated yields (for details, see Kromer et al. 2010), we mapped the abundance and density structure of the SN ejecta at the end $(t=100 \mathrm{~s})$ of the hydrodynamic simulations (by which point homologous expansion is a good approximation, e.g., Röpke 2005) to a $200^{3}$ Cartesian grid. The resulting ejecta structure is shown in Fig. 4. We note the asymmetry of the model, with the ejecta in the upper hemisphere extending to much higher velocities. This asymmetric extent of the ejecta in velocity space is a natural consequence of the single spot near-surface lit detonation (Chamulak et al. 2012). 


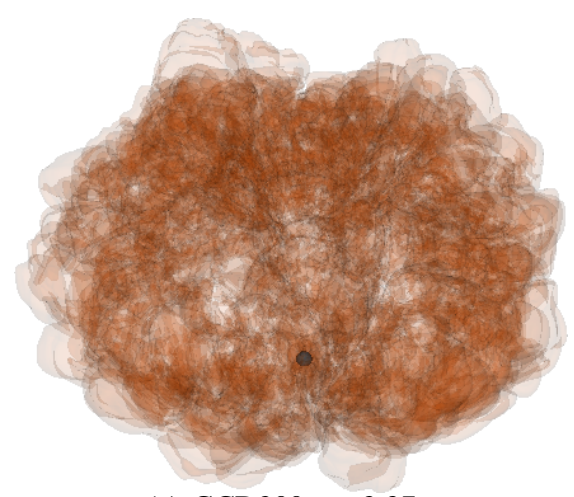

(a) GCD200, $\mathrm{t}=2.37 \mathrm{~s}$

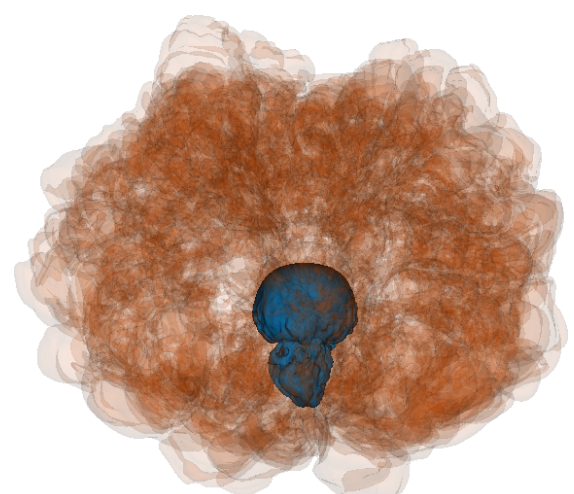

(d) GCD200, t $=2.7 \mathrm{~s}$

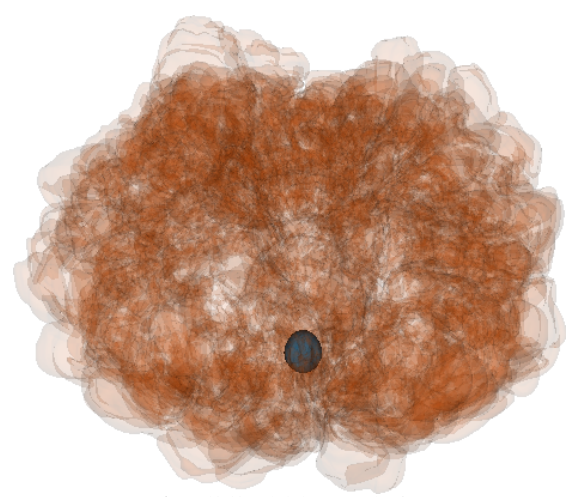

(b) GCD200, t $=2.4 \mathrm{~s}$

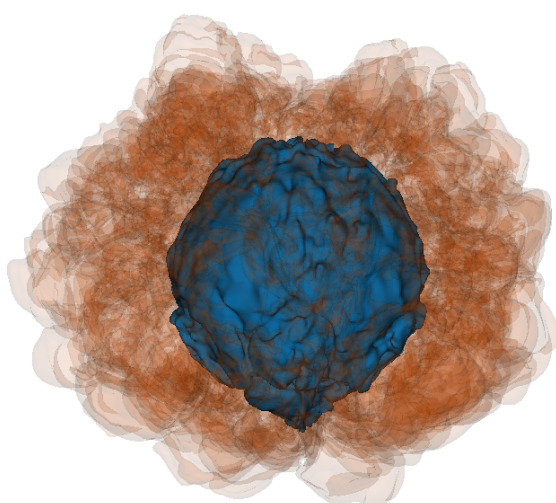

(e) GCD200, $\mathrm{t}=3.9 \mathrm{~s}$

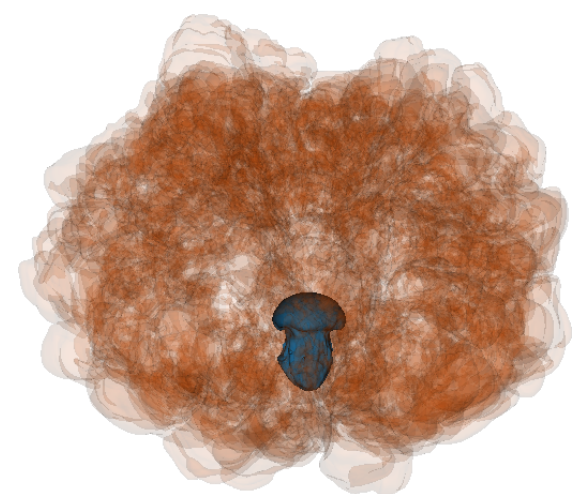

(c) GCD200, t $=2.5 \mathrm{~s}$

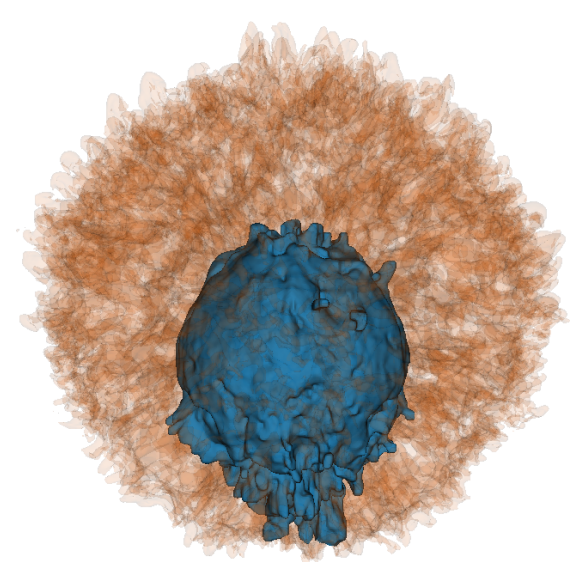

(f) $\mathrm{GCD} 200, \mathrm{t}=60 \mathrm{~s}$

Fig. 2. Evolution of the GCD200 model for six different instances of time. The deflagration level set is shown in red and the detonation level set in blue. Since the deflagration ashes almost completely surround the detonation, we have rendered the deflagration level set semi-transparent to allow visualizing the enclosed detonation front. The spatial scales in the plots are $1.23 \times 10^{10} \mathrm{~cm}, 1.25 \times 10^{10} \mathrm{~cm}, 1.33 \times 10^{10} \mathrm{~cm}, 1.47 \times 10^{10} \mathrm{~cm}$, $2.46 \times 10^{10} \mathrm{~cm}$, and $1.59 \times 10^{12} \mathrm{~cm}$ for panels a) to f), respectively.

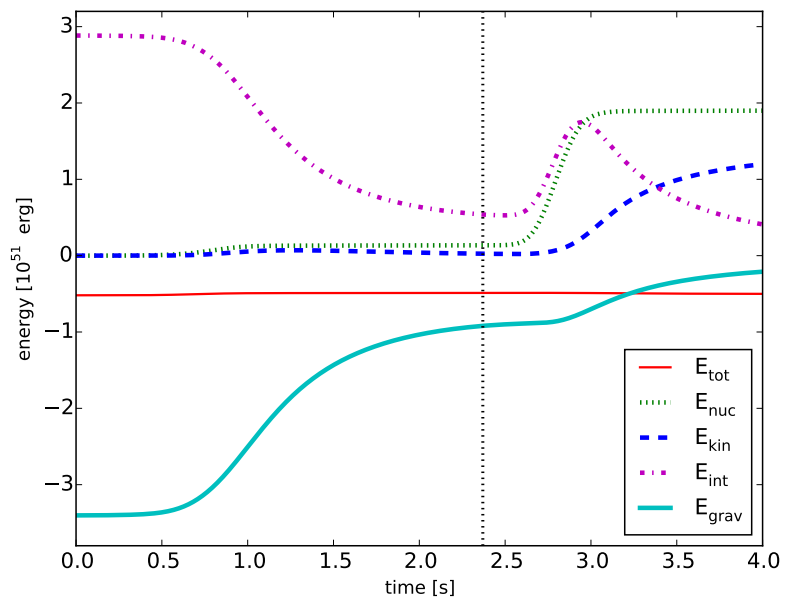

(a)

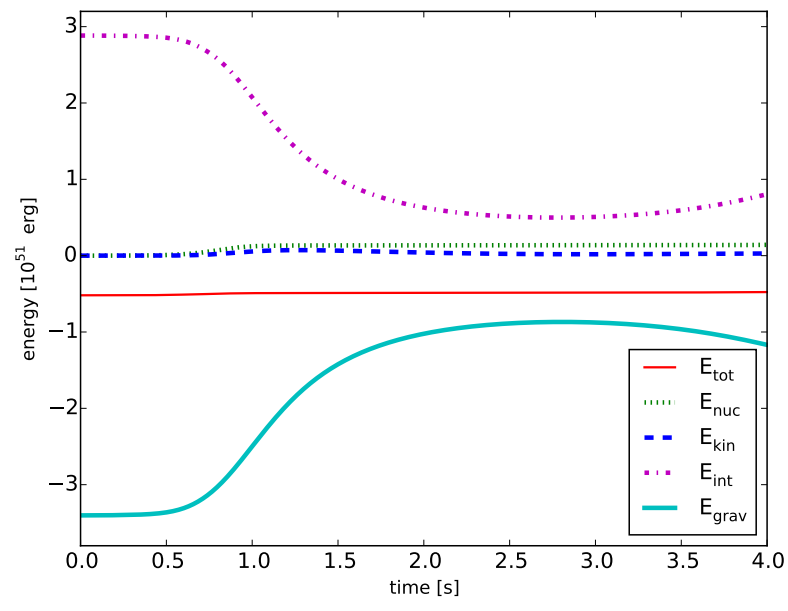

(b)

Fig. 3. Temporal evolution of $E_{\mathrm{tot}}, E_{\mathrm{nuc}}, E_{\mathrm{kin}}, E_{\mathrm{int}}$, and $E_{\mathrm{grav}}$. The left panel shows model GCD200. The vertical line marks the time at which the detonation is initialised. The right panel is for the corresponding pure deflagration case when no detonation is initiated.

The direction of travel of the detonation points into the star on one hemisphere (with a component against the direction of expansion; along gravity) and points out of the star on the other hemisphere (with a component along the direction of expansion; against gravity), which leads to the systematic asymmetry of the ejecta.
The total mass of ${ }^{56} \mathrm{Ni}$ produced is $0.74 M_{\odot}$, hence we obtain a bright explosion. This ${ }^{56} \mathrm{Ni}$ mass is very close to the $0.78 M_{\odot}$ of ${ }^{56} \mathrm{Ni}$ determined for SN $1991 \mathrm{~T}$ by Sasdelli et al. (2014). Using the method of abundance tomography (Stehle et al. 2005), Sasdelli et al. (2014) also found that they required three per cent of ${ }^{56} \mathrm{Ni}$ by mass at velocities above $12500 \mathrm{~km} \mathrm{~s}^{-1}$ 
Table 1. Stable isotopes (asymptotic values) of model GCD200 in solar masses.

\begin{tabular}{lllc}
\hline \hline${ }^{\mathrm{Isotope}}$ & $\begin{array}{c}\text { Mass } \\
{[M \odot]}\end{array}$ & ${ }^{\text {Isotope }}$ & $\begin{array}{c}\text { Mass } \\
{[M \odot}\end{array}$ \\
\hline & & $0.00 \mathrm{E}+00$ \\
\hline${ }^{1} \mathrm{H}$ & $6.19 \mathrm{E}-14$ & ${ }^{2} \mathrm{H}$ & 0.00 \\
${ }^{3} \mathrm{He}$ & $0.00 \mathrm{E}+00$ & ${ }^{4} \mathrm{He}$ & $1.29 \mathrm{E}-03$ \\
${ }^{6} \mathrm{Li}$ & $5.55 \mathrm{E}-21$ & ${ }^{7} \mathrm{Li}$ & $1.61 \mathrm{E}-19$ \\
${ }^{9} \mathrm{Be}$ & $0.00 \mathrm{E}+00$ & ${ }^{10} \mathrm{~B}$ & $7.92 \mathrm{E}-16$ \\
${ }^{11} \mathrm{~B}$ & $4.40 \mathrm{E}-12$ & ${ }^{12} \mathrm{C}$ & $1.88 \mathrm{E}-02$ \\
${ }^{13} \mathrm{C}$ & $6.62 \mathrm{E}-08$ & ${ }^{14} \mathrm{~N}$ & $1.49 \mathrm{E}-05$ \\
${ }^{15} \mathrm{~N}$ & $2.04 \mathrm{E}-08$ & ${ }^{16} \mathrm{O}$ & $1.13 \mathrm{E}-01$ \\
${ }^{17} \mathrm{O}$ & $1.98 \mathrm{E}-06$ & ${ }^{18} \mathrm{O}$ & $2.54 \mathrm{E}-08$ \\
${ }^{19} \mathrm{~F}$ & $2.45 \mathrm{E}-10$ & ${ }^{20} \mathrm{Ne}$ & $6.83 \mathrm{E}-03$ \\
${ }^{21} \mathrm{Ne}$ & $1.64 \mathrm{E}-06$ & ${ }^{22} \mathrm{Ne}$ & $6.73 \mathrm{E}-04$ \\
${ }^{23} \mathrm{Na}$ & $1.16 \mathrm{E}-04$ & ${ }^{24} \mathrm{Mg}$ & $1.96 \mathrm{E}-02$ \\
${ }^{25} \mathrm{Mg}$ & $1.68 \mathrm{E}-04$ & ${ }^{26} \mathrm{Mg}$ & $2.46 \mathrm{E}-04$ \\
${ }^{27} \mathrm{Al}$ & $1.00 \mathrm{E}-03$ & ${ }^{28} \mathrm{Si}$ & $2.52 \mathrm{E}-01$ \\
${ }^{29} \mathrm{Si}$ & $1.08 \mathrm{E}-03$ & ${ }^{30} \mathrm{Si}$ & $2.71 \mathrm{E}-03$ \\
${ }^{31} \mathrm{P}$ & $5.39 \mathrm{E}-04$ & ${ }^{32} \mathrm{~S}$ & $1.02 \mathrm{E}-01$ \\
${ }^{33} \mathrm{~S}$ & $2.36 \mathrm{E}-04$ & ${ }^{34} \mathrm{~S}$ & $2.82 \mathrm{E}-03$ \\
${ }^{36} \mathrm{~S}$ & $2.63 \mathrm{E}-07$ & ${ }^{35} \mathrm{Cl}$ & $1.29 \mathrm{E}-04$ \\
${ }^{37} \mathrm{Cl}$ & $2.15 \mathrm{E}-05$ & ${ }^{36} \mathrm{Ar}$ & $1.69 \mathrm{E}-02$ \\
${ }^{38} \mathrm{Ar}$ & $1.21 \mathrm{E}-03$ & ${ }^{40} \mathrm{Ar}$ & $8.37 \mathrm{E}-09$ \\
${ }^{39} \mathrm{~K}$ & $6.58 \mathrm{E}-05$ & ${ }^{41} \mathrm{~K}$ & $3.58 \mathrm{E}-06$ \\
${ }^{40} \mathrm{Ca}$ & $1.49 \mathrm{E}-02$ & ${ }^{42} \mathrm{Ca}$ & $2.45 \mathrm{E}-05$ \\
${ }^{43} \mathrm{Ca}$ & $2.91 \mathrm{E}-08$ & ${ }^{44} \mathrm{Ca}$ & $9.28 \mathrm{E}-06$ \\
${ }^{46} \mathrm{Ca}$ & $2.58 \mathrm{E}-11$ & ${ }^{48} \mathrm{Ca}$ & $9.40 \mathrm{E}-16$ \\
${ }^{45} \mathrm{Sc}$ & $1.34 \mathrm{E}-07$ & ${ }^{46} \mathrm{Ti}$ & $1.32 \mathrm{E}-05$ \\
${ }^{47} \mathrm{Ti}$ & $4.58 \mathrm{E}-07$ & ${ }^{48} \mathrm{Ti}$ & $3.09 \mathrm{E}-04$ \\
${ }^{49} \mathrm{Ti}$ & $2.31 \mathrm{E}-05$ & ${ }^{50} \mathrm{Ti}$ & $1.91 \mathrm{E}-10$ \\
${ }^{50} \mathrm{~V}$ & $1.30 \mathrm{E}-09$ & ${ }^{51} \mathrm{~V}$ & $7.91 \mathrm{E}-05$ \\
${ }^{50} \mathrm{Cr}$ & $3.31 \mathrm{E}-04$ & ${ }^{52} \mathrm{Cr}$ & $7.29 \mathrm{E}-03$ \\
${ }^{53} \mathrm{Cr}$ & $7.40 \mathrm{E}-04$ & ${ }^{54} \mathrm{Cr}$ & $1.25 \mathrm{E}-07$ \\
${ }^{55} \mathrm{Mn}$ & $4.91 \mathrm{E}-03$ & ${ }^{54} \mathrm{Fe}$ & $2.96 \mathrm{E}-02$ \\
${ }^{56} \mathrm{Fe}$ & $7.43 \mathrm{E}-01$ & ${ }^{57} \mathrm{Fe}$ & $1.84 \mathrm{E}-02$ \\
${ }^{58} \mathrm{Fe}$ & $7.04 \mathrm{E}-08$ & ${ }^{59} \mathrm{Co}$ & $3.45 \mathrm{E}-04$ \\
${ }^{58} \mathrm{Ni}$ & $3.74 \mathrm{E}-02$ & ${ }^{60} \mathrm{Ni}$ & $1.50 \mathrm{E}-03$ \\
${ }^{61} \mathrm{Ni}$ & $6.47 \mathrm{E}-05$ & ${ }^{62} \mathrm{Ni}$ & $5.24 \mathrm{E}-04$ \\
${ }^{64} \mathrm{Ni}$ & $1.10 \mathrm{E}-13$ & ${ }^{63} \mathrm{Cu}$ & $3.08 \mathrm{E}-07$ \\
${ }^{65} \mathrm{Cu}$ & $5.85 \mathrm{E}-08$ & ${ }^{64} \mathrm{Zn}$ & $9.31 \mathrm{E}-07$ \\
${ }^{66} \mathrm{Zn}$ & $1.44 \mathrm{E}-06$ & ${ }^{67} \mathrm{Zn}$ & $7.72 \mathrm{E}-10$ \\
${ }^{68} \mathrm{Zn}$ & $4.90 \mathrm{E}-10$ & ${ }^{70} \mathrm{Zn}$ & $4.81 \mathrm{E}-24$ \\
${ }^{71} \mathrm{Ga}-15$ & $7.24 \mathrm{E}-16$ \\
& & & \\
\hline
\end{tabular}

to match the spectral time evolution of SN 1991T. The earlier work by Mazzali et al. (1995) found that the outer layers of SN 1991T are dominated by IGEs, which would argue in favour of a GCD origin. However, according to Sasdelli et al. (2014), the large amount of high-velocity ${ }^{56} \mathrm{Ni}$ found by Mazzali et al. (1995) is a result of a larger assumed distance modulus ( $\mu=30.65$ ); Sasdelli et al. (2014) preferred $\mu=30.57$, which leads to improved spectral fits at all photospheric epochs. To facilitate the comparison with their abundance tomography results (Figs. 5 and 7 of Sasdelli et al. 2014), we show the chemical ejecta structure in velocity space along two lines of sight ( $\pm z$-axes, see Fig. 5).
Table 2. Radioactive isotopes of model GCD200 at $t=100 \mathrm{~s}$ in solar masses.

\begin{tabular}{lclc}
\hline \hline Isotope & $\begin{array}{c}\text { Mass } \\
{\left[M_{\odot}\right]}\end{array}$ & Isotope & $\begin{array}{c}\text { Mass } \\
{\left[M_{\odot}\right]}\end{array}$ \\
\hline${ }^{14} \mathrm{C}$ & $1.14 \mathrm{E}-05$ & ${ }^{22} \mathrm{Na}$ & $9.39 \mathrm{E}-09$ \\
${ }^{26} \mathrm{Al}$ & $1.29 \mathrm{E}-06$ & ${ }^{32} \mathrm{Si}$ & $2.18 \mathrm{E}-08$ \\
${ }^{32} \mathrm{P}$ & $5.36 \mathrm{E}-07$ & ${ }^{33} \mathrm{P}$ & $3.98 \mathrm{E}-07$ \\
${ }^{35} \mathrm{~S}$ & $5.25 \mathrm{E}-07$ & ${ }^{36} \mathrm{Cl}$ & $6.50 \mathrm{E}-07$ \\
${ }^{37} \mathrm{Ar}$ & $2.08 \mathrm{E}-05$ & ${ }^{39} \mathrm{Ar}$ & $7.73 \mathrm{E}-09$ \\
${ }^{40} \mathrm{~K}$ & $3.86 \mathrm{E}-08$ & ${ }^{41} \mathrm{Ca}$ & $3.57 \mathrm{E}-06$ \\
${ }^{44} \mathrm{Ti}$ & $9.26 \mathrm{E}-06$ & ${ }^{48} \mathrm{~V}$ & $5.55 \mathrm{E}-08$ \\
${ }^{49} \mathrm{~V}$ & $2.14 \mathrm{E}-07$ & ${ }^{48} \mathrm{Cr}$ & $3.09 \mathrm{E}-04$ \\
${ }^{49} \mathrm{Cr}$ & $2.29 \mathrm{E}-05$ & ${ }^{51} \mathrm{Cr}$ & $3.65 \mathrm{E}-06$ \\
${ }^{51} \mathrm{Mn}$ & $7.55 \mathrm{E}-05$ & ${ }^{52} \mathrm{Mn}$ & $3.42 \mathrm{E}-06$ \\
${ }^{53} \mathrm{Mn}$ & $4.82 \mathrm{E}-05$ & ${ }^{54} \mathrm{Mn}$ & $1.25 \mathrm{E}-07$ \\
${ }^{52} \mathrm{Fe}$ & $7.23 \mathrm{E}-03$ & ${ }^{53} \mathrm{Fe}$ & $6.92 \mathrm{E}-04$ \\
${ }^{55} \mathrm{Fe}$ & $1.35 \mathrm{E}-04$ & ${ }^{59} \mathrm{Fe}$ & $1.91 \mathrm{E}-15$ \\
${ }^{60} \mathrm{Fe}$ & $7.93 \mathrm{E}-18$ & ${ }^{55} \mathrm{Co}$ & $4.77 \mathrm{E}-03$ \\
${ }^{56} \mathrm{Co}$ & $2.40 \mathrm{E}-05$ & ${ }^{57} \mathrm{Co}$ & $2.77 \mathrm{E}-05$ \\
${ }^{58} \mathrm{Co}$ & $6.64 \mathrm{E}-08$ & ${ }^{60} \mathrm{Co}$ & $9.99 \mathrm{E}-13$ \\
${ }^{56} \mathrm{Ni}$ & $7.42 \mathrm{E}-01$ & ${ }^{57} \mathrm{Ni}$ & $1.84 \mathrm{E}-02$ \\
${ }^{59} \mathrm{Ni}$ & $6.93 \mathrm{E}-05$ & ${ }^{63} \mathrm{Ni}$ & $7.71 \mathrm{E}-14$ \\
${ }^{62} \mathrm{Zn}$ & $5.24 \mathrm{E}-04$ & ${ }^{65} \mathrm{Zn}$ & $3.53 \mathrm{E}-10$ \\
${ }^{65} \mathrm{Ga}$ & $3.97 \mathrm{E}-08$ & ${ }^{68} \mathrm{Ge}$ & $4.70 \mathrm{E}-10$ \\
\hline
\end{tabular}

The high-velocity IGEs pose a problem to our model (when compared to SN 1991T): the shell of deflagration ashes has an IGE content of $\sim 40$ per cent by mass in almost every direction, which strongly contradicts Sasdelli et al. (2014). Furthermore, again in contrast to the result of Sasdelli et al. (2014), the GCD model does not predict a low-velocity core dominated by stable Fe. As expected, neutron-rich stable Fe-isotopes are produced at the highest densities where electron captures lower the electron fraction. For the GCD model, however, these products of the high-density burning unavoidably rise towards the surface due to buoyancy, even for the case of central igntion (Malone et al. 2014). A consequence of the buoyancy of the hot deflagration ashes is that the stable IGEs end up predominantly at the highest velocities (see Fig. 4 and top panel of Fig. 5). In contrast, the low-velocity core is dominated by ${ }^{56} \mathrm{Ni}$, which is synthesised in the detonation after the WD has expanded to lower central density. The high-velocity deflagration ashes are therefore a characteristic feature of the underlying explosion mechanism of the model, which indeed relies on the convergent flow of the deflagration products on the surface of the WD to trigger the detonation. The distribution of the intermediate mass elements, on the other hand, generally agrees quite well for a few prominent species such as $\mathrm{O}, \mathrm{S}$, and $\mathrm{Si}$.

There is, however, more intriguing agreement between the special line of sight along the negative $z$-axis (bottom panel of Fig. 5) and several features of the abundance tomography results from Sasdelli et al. (2014). Since the deflagration ashes failed to fully surround the region where the detonation initiated, this particular line of sight is less contaminated by high-velocity deflagration ashes, which improves the comparison. Si and $\mathrm{S}$ for example peak between $10000 \mathrm{~km} \mathrm{~s}^{-1}$ and $13000 \mathrm{~km} \mathrm{~s}^{-1}$, $\mathrm{O}$ dominates the outer ejecta at high velocity $\left(>12500 \mathrm{~km} \mathrm{~s}^{-1}\right)$, and ${ }^{56} \mathrm{Ni}$ is present at high velocity at the few per cent level, all in good agreement with the tomography results of Sasdelli et al. (2014). The discrepancy concerning the absence of appreciable 


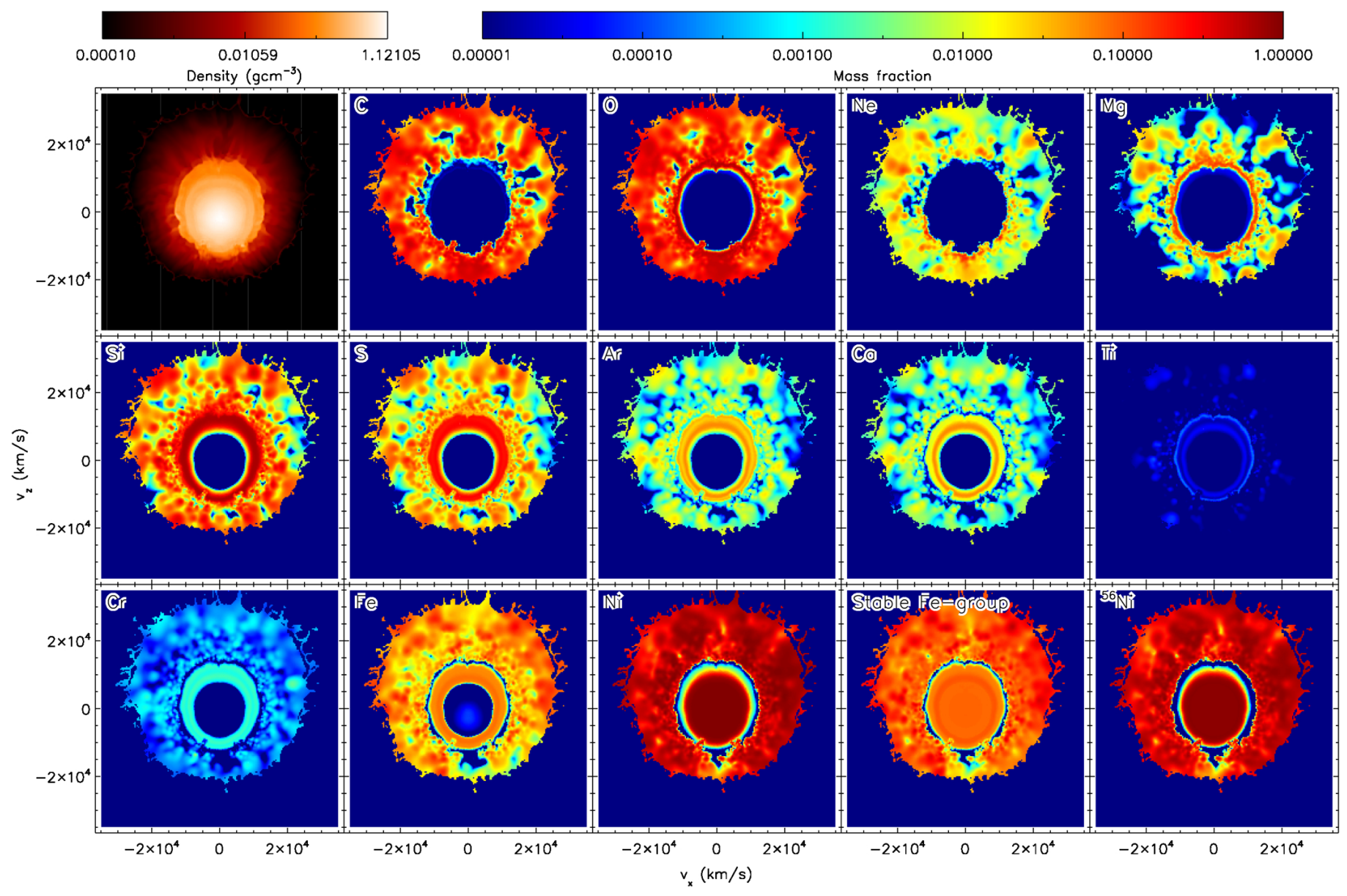

Fig. 4. Final ejecta structure of model GCD200 in asymptotic velocity space at $100 \mathrm{~s}$. We show the density (top left panel) and the mass fractions of select species in a slice through the midplane of the simulation volume along the $x$-and $z$-axes (detonation ignition occurred close to the negative $z$-axis).

low-velocity stable Fe however remains, and $\mathrm{C}$ is also much more abundant at high velocity in the model than in the tomography results. Still, this is the line of sight where the model resembles the observationally inferred abundance stratification the most. However, there is only a small solid angle of $\sim 0.38 \mathrm{sr}$ (corresponding to a cone of half-opening angle of $\lesssim 20^{\circ}$ ) where the deflagration ashes are nearly absent from the surface (see Fig. 4). This strongly argues against an identification of this special viewing direction with SNe 91T: a view of the supernova from a line of sight that intersects the deflagration ashes is more than thirty times more likely, and those viewing angles are lacking potential SN counterparts. In the next section, we present the results of our radiative transfer calculation for the GCD200 explosion model and compare the synthetic observables to SN 1991T.

\section{Synthetic observables}

To obtain synthetic spectra and light curves for the GCD200 model, we used the time-dependent 3D Monte Carlo radiative transfer code ARTIS (Kromer \& Sim 2009; Sim 2007). For our radiative transfer simulation, we remapped the ejecta structure to a $50^{3}$ grid and followed the propagation of $10^{8}$ photon packets for 111 logarithmically spaced time-steps between 2 and $120 \mathrm{~d}$ after explosion. To reduce the computational costs, a grey approximation, as discussed by Kromer \& Sim (2009), was used in optically thick cells, and for $t<3 \mathrm{~d}$ local thermodynamic equilibrium was assumed. We used the atomic data set described in Gall et al. (2012). Synthetic spectra for 121 equally sized viewing-angle bins covering the full solid angle were extracted on a 1000-bin logarithmic wavelength grid spanning a range between 600 and $30000 \AA$.

On large scales, the GCD200 model is roughly symmetric under rotation about the axis defined by the centre of the star and the location of the initial deflagration bubble (the $z$-axis in Fig. 4). Thus, we can integrate the synthetic spectra over the equatorial angle with respect to the $z$-axis, leaving 11 equally sized viewing-angle bins in $\cos \theta$. Figure 6 shows snapshots of the spectral evolution of our model from $7.4 \mathrm{~d}$ to $33.6 \mathrm{~d}$ past explosion.

At early times $(t \lesssim 25 \mathrm{~d})$, the model spectra show a prominent viewing-angle sensitivity in the ultraviolet (UV) and blue wavelength regions where the flux level decreases from $\cos \theta=-1$ to $\cos \theta=1$ by up to a factor 10 at certain wavelengths. This is due to the asymmetric distribution of the IGE-rich deflagration ashes: on the deflagration ignition side $(\cos \theta=1)$ IGEs are abundant up to $\sim 30000 \mathrm{~km} \mathrm{~s}^{-1}$, while the ejecta extend only to $\sim 20000 \mathrm{~km} \mathrm{~s}^{-1}$ on the far side $(\cos \theta=-1)$ where the detonation ignites (see Fig. 4). This leads to a significant reduction of IGE line blanketing from $\cos \theta=1$ to $\cos \theta=-1$ and thus an enhancement of the flux in the UV and blue regions when viewed from the detonation side. Redder wavelengths $(\lambda \gtrsim 5000 \AA)$ are not affected by line blanketing from IGEs and thus show no prominent viewing-angle sensitivity. At later times $(t \gtrsim 25 \mathrm{~d})$, the outer layers become optically thin and the viewing-angle sensitivity in the blue bands decreases. The remaining viewing-angle 


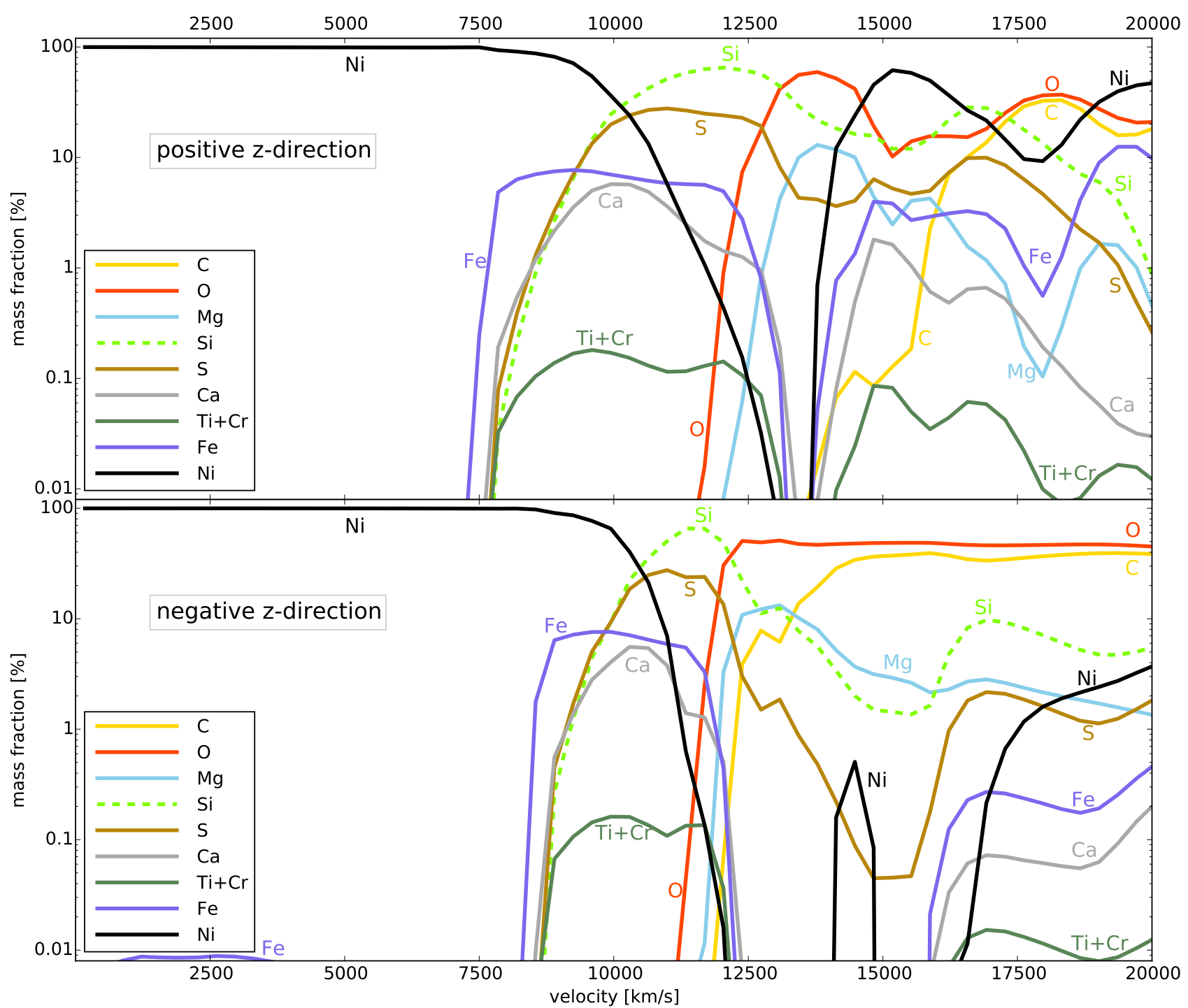

Fig. 5. Final ejecta structure of model GCD200 along the positive (top) and negative (bottom) $z$-axis in asymptotic velocity space at $100 \mathrm{~s}$. Detonation ignition occurred close to the negative $z$-axis. The relative absence of high-velocity IGE in this direction is a reflection of the lack of deflagration ashes (see Fig. 4). The profiles along the $\pm x$-axis and $\pm y$-axis qualitatively resemble the top panel.

sensitivity is mainly due to the off-centre structure of the detonation ashes (see Fig. 4).

The same behaviour can also be observed in the synthetic light curves (Fig. 7), which show a strong variation around the peak in the $U$ and $B$ bands. For $U$, the peak magnitudes vary between -18.9 and -20.1 mag with rise times between 16.8 and $17.5 \mathrm{~d}$. The $B$-band light curves peak between 19.2 and $19.9 \mathrm{~d}$ at magnitudes between -19.3 and -19.8 mag. In contrast, we do not observe any significant variation in $V, R$, and $I$. After maximum the viewing angle sensitivity also decreases in the bluer bands.

The $U$ - and $B$-band light curves show an additional effect at very early epochs $(t \lesssim 5 \mathrm{~d})$. At these epochs $\gamma$-rays from ${ }^{56} \mathrm{Ni}$ decay in the deflagration ashes are still trapped, leading to additional surface heating and an enhanced flux in the UV. This causes a decline in the $U$-band light curves at the earliest epochs and a plateau-like behaviour in the $B$ band.

Figure 7 also shows light curves for three observed SNe Ia: the luminous SN 1991T (Lira et al. 1998) as well as SN 2004eo (Pastorello et al. 2007a) and SN 2005cf (Pastorello et al. 2007b), which are representative for the class of normal SNe Ia. Our model light curves are not a particularly good fit to any of these objects. Compared to normal SNe Ia, the model light curves are clearly too bright. This is expected because the ${ }^{56} \mathrm{Ni}$ mass of our model $\left(0.74 M_{\odot}\right)$ is significantly higher than the typical range of 0.4 to $0.6 M_{\odot}$ inferred for most SNe Ia (Scalzo et al. 2014). Compared to SN 1991T, an equatorial viewing angle $(\cos \theta=0)$ is in reasonable agreement with the observed $B$ - and $V$-band light curves for $t \lesssim 10 \mathrm{~d}$ and $t \gtrsim 30 \mathrm{~d}$. However, around the peak a viewing angle close to the deflagration ignition side agrees better with the observed $B$-band light curve of SN 1991T, while in the $V$-band our model is significantly too bright for all viewing angles.

Our model spectra also fail to convincingly match observed $\mathrm{SN}$ Ia spectra. Compared to normal $\mathrm{SNe}$ Ia, the line features associated with intermediate-mass elements (IMEs) are slightly too weak. Si II $\lambda 5972$, for example, is very weak in the model spectra, indicating relatively high ionisation and temperature of the ejecta. This is corroborated by the presence of absorption features at $\sim 4200$ and $4900 \AA$ that are attributed to Fe III. Compared 

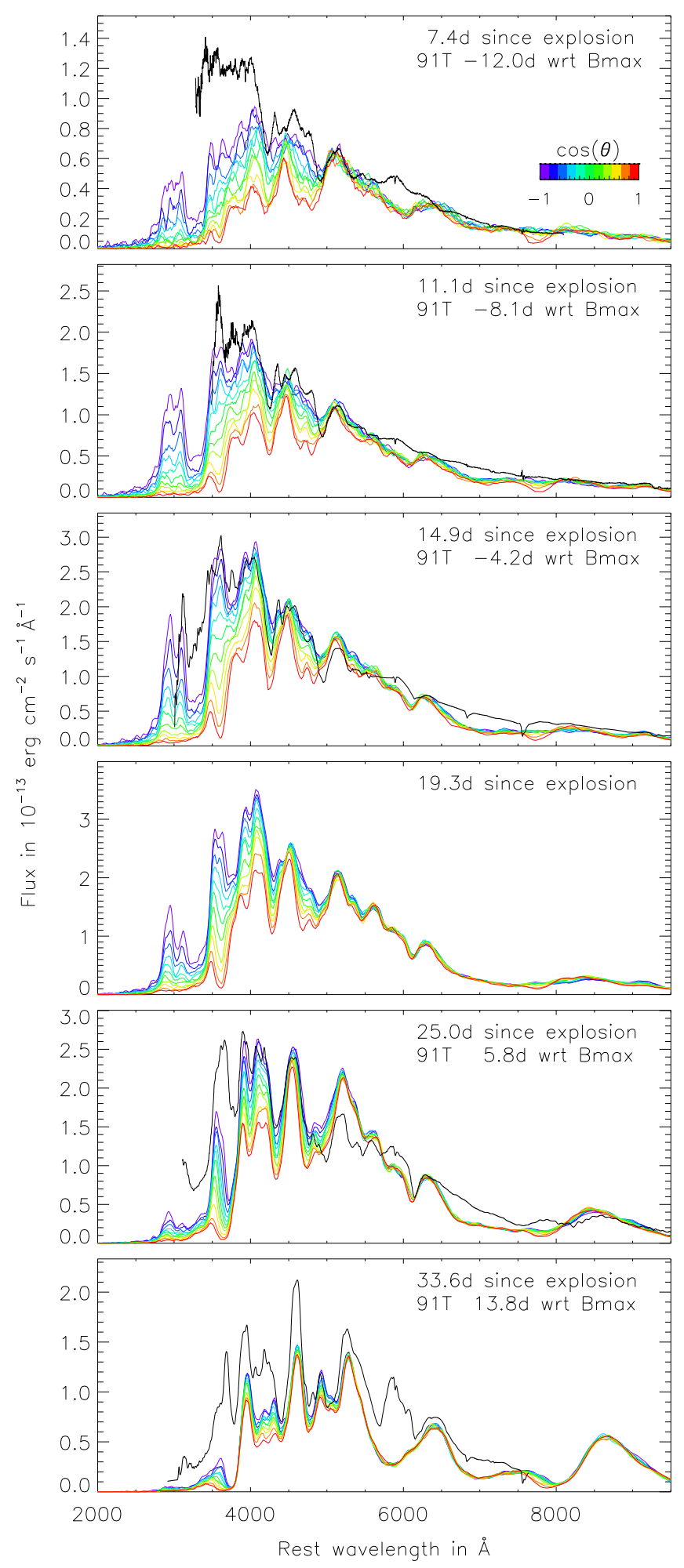

Fig. 6. Time sequence of synthetic spectra for our model (lines of different colours correspond to different viewing angles as indicated by the colour bar). The snapshot at $19.3 \mathrm{~d}$ corresponds roughly to $B$-band maximum in the model, which occurs between 19.2 and $19.9 \mathrm{~d}$ depending on the viewing angle. The synthetic spectra have been smoothed with a Savitzky-Golay filter to reduce Monte Carlo noise. For comparison we overplot observed spectra of SN 1991T at corresponding epochs (black, the flux calibration has been checked against the photometry and adjusted when necessary). The observed spectra have been de-reddened $(E(B-V)=0.13$, Phillips et al. 1992) and de-redshifted $(z=0.006059$, from interstellar $\mathrm{Na})$. We assumed a distance modulus of 30.76 to SN 1991T (Saha et al. 2006).

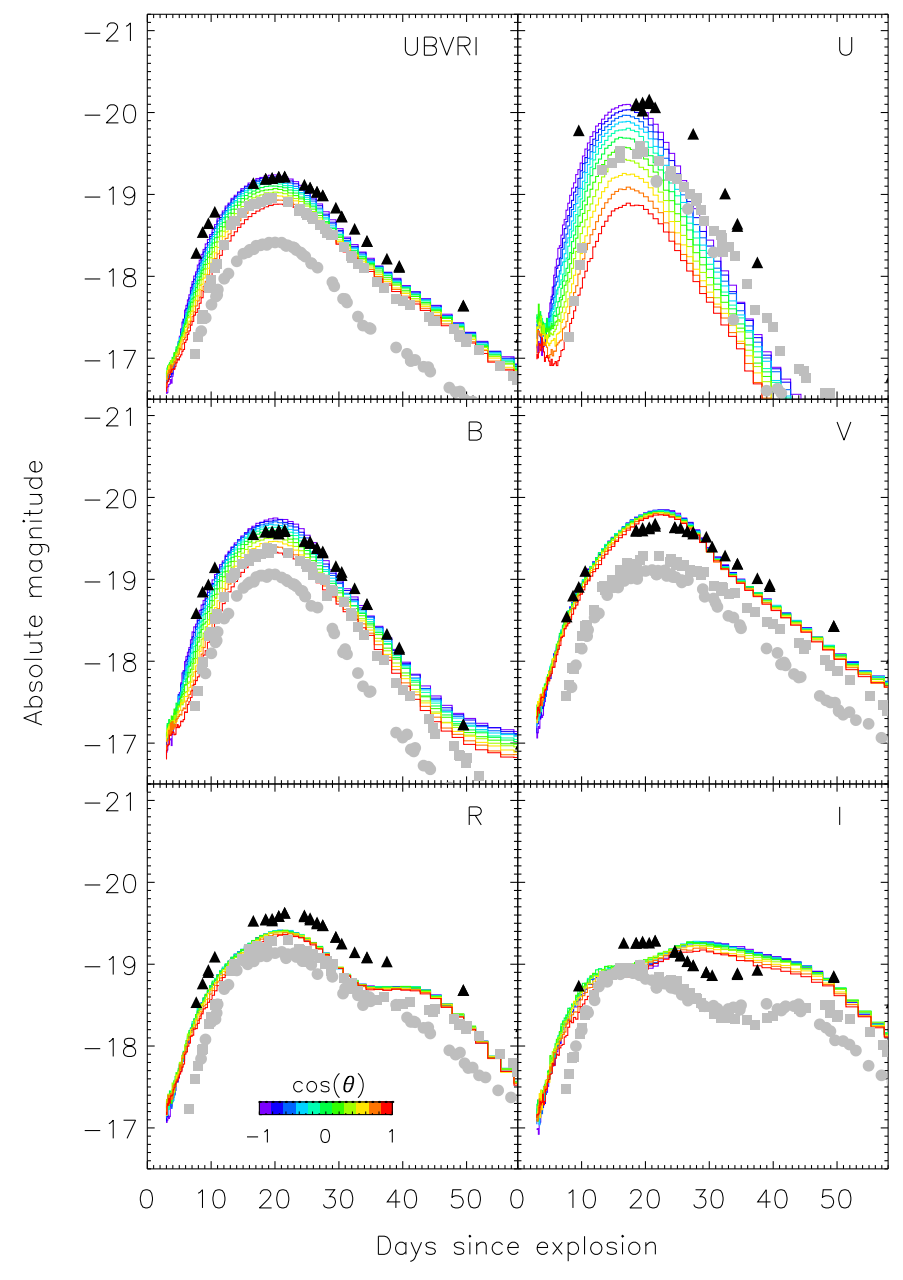

Fig. 7. Synthetic broadband light curves for our model. Lines of different colour correspond to different viewing angles as indicated by the colour bar. Overplotted are observed light curves for the normal SNe Ia 2004eo (grey circles) and 2005cf (grey squares), and for the overluminous SN 1991T (black triangles). For the comparison we assume a $B$-band rise time of $19.6 \mathrm{~d}$, which corresponds to the average $B$-band rise time of our model.

to SN 1991T, there is no individual viewing angle that provides good agreement over the full wavelength range and multiple epochs (see Fig. 6). Although relatively weak, the IME features of our model are still too strong at pre-maximum epochs when SN $1991 \mathrm{~T}$ shows no clear signs of the Ca II H\&K lines and S II $\lambda$ 5624. Si II $\lambda 6355$ develops in SN 1991T a week before maximum, but it is also weaker than in our model.

\section{Summary and conclusions}

The purpose of this work is to calculate the generic observables of a GCD model if a detonation occurs, not to settle the question whether a detonation can form in the first place. Deflagrations that are ignited closer to the centre lead to more burning and expansion, which leads to a weaker collision of the deflagration ashes at the opposite point of break-out. To obtain detonation conditions and a subsequent GCD SN explosion, we ignited a near- $M_{\mathrm{Ch}}$ WD outside the preferred range predicted by numerical simulations (Nonaka et al. 2012) and chose less stringent critical detonation conditions compared to previous works (cf. Röpke et al. 2007). 
Our high-resolution model $\left(512^{3}\right)$ was ignited $(200 \mathrm{~km})$ and fullfilled our deliberately optimistic critical detonation conditions, and we initialised a detonation and followed the explosion until homologous expansion. Given the large offset, relatively little mass burned in the deflagration, which resulted in a relatively compact WD at the instant of detonation initiation. This means that GCD explosions are automatically linked to the brighter end of the SN Ia distribution. If the WD were ignited closer to the centre, we would obtain a stronger deflagration, resulting in stronger expansion, no detonation, and a sub-luminous SN 2002cx-like event (Jordan et al. 2012b; Kromer et al. 2013).

By post-processing one million tracer particles with a nuclear reaction network, we determined the detailed nucleosynthesis in the explosion. A high-velocity outer shell of deflagration ashes rich in ${ }^{56} \mathrm{Ni}$ and stable iron group isotopes such as ${ }^{54} \mathrm{Fe}$ and ${ }^{58} \mathrm{Ni}$ is a generic and robust prediction for this class of models. This shell of IGE-rich deflagration ashes exhibits a gradient in its extent in velocity space, decreasing in extent from the deflagration-ignition side to the far side where the detonationinitiation is located.

We used the resulting three-dimensional composition structure of the explosion ejecta as input for a Monte Carlo radiative transfer calculation with the ARTIS code to obtain time-dependent spectra and light curves for the GCD200 model. Comparing these synthetic observables to $\mathrm{SN}$ Ia data, we conclude that this GCD model cannot explain any of the more common sub-classes of SNe Ia. In particular, it fails to explain SN 1991T-like events, a class of SNe that owing to their brightness and early high-velocity Fe-features appeared to be the most natural candidate (Fisher \& Jumper 2015). First, the single-spot ignition of the deflagration on one side of the star and the initiation of the detonation on the opposite side naturally leads to an asymmetric extent of the shell of deflagration ashes in velocity space, extending to much higher velocity on the side where the deflagration was ignited. This in turn leads to a strong viewing-angle dependence for the synthetic spectra and light curves, most pronounced in the bluer bands at early times, which disagrees with observations. Since the ejecta asymmetry is a generic feature of GCD explosions (Chamulak et al. 2012), the resulting viewing angle sensitivity is generic as well. Identification of SNe 91T with only the peculiar line of sight towards the location of the initiation of the detonation, which gives the best agreement, leads to the following problem: the other viewing angles characterised by high-velocity turbulently mixed deflagration ashes rich in ${ }^{56} \mathrm{Ni}$ and stable IGE statistically dominate and do not correspond to a known more common sub-class of SNe. Second, our model is too abundant in IGEs at high velocity and lacks the stable lowvelocity $\mathrm{Fe}$ inferred by Sasdelli et al. (2014) by abundance tomography methods. Third, although the overall flux-levels compare favourably, the spectral features do not. For example the Ca II H\&K lines, S II $\lambda$ 5624, and Si II $\lambda$ 6355, which are absent or very weak pre-maximum in SN 1991T, are too strong in our model for all lines of sight. Particularly the first two points are very reliable generic features of GCD explosion models. Overall, our results therefore suggest that GCD is probably not a good explosion model for SN 1991T. Whether more peculiar events can be explained by GCD events remains to be seen.

Acknowledgements. This work was supported by Australian Research Council Laureate Grant FL0992131, the Deutsche Forschungsgemeinschaft via the Transregional Collaborative Research Center TRR 33 "The Dark Universe", the Emmy Noether Program (RO 3676/1-1), the ARCHES prize of the German Ministry of Education and Research (BMBF), the graduate school "Theoretical Astrophysics and Particle Physics" at the University of Würzburg (GRK 1147) and the Excellence Cluster EXC 153 "Origin and Structure of the Universe".
S.A.S. acknowledges support from STFC grant ST/L000709/1. A.J.R. is thankful for funding provided by the Australian Research Council Centre of Excellence for All-sky Astrophysics (CAASTRO) through project number CE110001020. RP acknowledges support by the European Research Council under ERC-StG grant EXAGAL-308037. We also thank the DAAD/Go8 German-Australian exchange programme for travel support. S.T.O. acknowledges support from Studienstiftung des deutschen Volkes. The work of S.T.O., R.P., and F.K.R is supported by the Klaus Tschira Foundation. This research was supported by the Partner Time Allocation (Australian National University), the National Computational Merit Allocation and the Flagship Allocation Schemes of the NCI National Facility at the Australian National University. The authors also gratefully acknowledge the Gauss Centre for Supercomputing (GCS) for providing computing time through the John von Neumann Institute for Computing (NIC) on the GCS share of the supercomputer JUQUEEN (Stephan \& Docter 2015) at Jülich Supercomputing Centre (JSC). GCS is the alliance of the three national supercomputing centres HLRS (Universität Stuttgart), JSC (Forschungszentrum Jülich), and LRZ (Bayerische Akademie der Wissenschaften), funded by the German Federal Ministry of Education and Research (BMBF) and the German State Ministries for Research of Baden-Württemberg (MWK), Bayern (StMWFK), and Nordrhein-Westfalen (MIWF).

\section{References}

Aspden, A. J., Bell, J. B., Dong, S., \& Woosley, S. E. 2011, ApJ, 738, 94 Asplund, M., Grevesse, N., Sauval, A. J., \& Scott, P. 2009, ARA\&A, 47, 481 Blondin, S., Matheson, T., Kirshner, R. P., et al. 2012, AJ, 143, 126 Branch, D., Fisher, A., \& Nugent, P. 1993, AJ, 106, 2383

Bravo, E., \& Martínez-Pinedo, G. 2012, Phys. Rev. C, 85, 055805 Chamulak, D. A., Meakin, C. A., Seitenzahl, I. R., \& Truran, J. W. 2012, ApJ, 744, 27

Colella, P., \& Woodward, P. R. 1984, J. Comput. Phys., 54, 174

Cyburt, R. H., Amthor, A. M., Ferguson, R., et al. 2010, ApJS, 189, 240 Filippenko, A. V., Richmond, M. W., Matheson, T., et al. 1992, ApJ, 384, L15

Fink, M., Röpke, F. K., Hillebrandt, W., et al. 2010, A\&A, 514, A53

Fink, M., Kromer, M., Seitenzahl, I. R., et al. 2014, MNRAS, 438, 1762

Fisher, A., Branch, D., Hatano, K., \& Baron, E. 1999, MNRAS, 304, 67

Fisher, R., \& Jumper, K. 2015, ApJ, 805, 150

Fryxell, B. A., Müller, E., \& Arnett, W. D. 1989, Hydrodynamics and nuclear burning, MPA Green Report 449 (Garching: Max-Planck-Institut für Astrophysik)

Gall, E. E. E., Taubenberger, S., Kromer, M., et al. 2012, MNRAS, 427, 994

Garcia-Senz, D., \& Woosley, S. E. 1995, ApJ, 454, 895

García-Senz, D., Cabezón, R. M., Domínguez, I., \& Thielemann, F. K. 2016, ApJ, 819, 132

Guillochon, J., Dan, M., Ramirez-Ruiz, E., \& Rosswog, S. 2010, ApJ, 709, L64

Hamuy, M., Trager, S. C., Pinto, P. A., et al. 2000, AJ, 120, 1479

Han, Z., \& Podsiadlowski, P. 2004, MNRAS, 350, 1301

Hillebrandt, W., Kromer, M., Röpke, F. K., \& Ruiter, A. J. 2013, Frontiers of Physics, 8, 116

Howell, D. A. 2001, ApJ, 554, L193

Iben, Jr., I., \& Tutukov, A. V. 1984, ApJS, 54, 335

Jeffery, D. J., Leibundgut, B., Kirshner, R. P., et al. 1992, ApJ, 397, 304

Jordan, G. C., Gupta, S. S., \& Meyer, B. S. 2003, Phys. Rev. C, 68, 065801

Jordan, IV, G. C., Fisher, R. T., Townsley, D. M., et al. 2008, ApJ, 681, 1448

Jordan, IV, G. C., Graziani, C., Fisher, R. T., et al. 2012a, ApJ, 759, 53

Jordan, IV, G. C., Perets, H. B., Fisher, R. T., \& van Rossum, D. R. 2012b, ApJ, 761, L23

Kasen, D., \& Plewa, T. 2007, ApJ, 662, 459

Kromer, M., \& Sim, S. A. 2009, MNRAS, 398, 1809

Kromer, M., Sim, S. A., Fink, M., et al. 2010, ApJ, 719, 1067

Kromer, M., Fink, M., Stanishev, V., et al. 2013, MNRAS, 429, 2287

Kuhlen, M., Woosley, S. E., \& Glatzmaier, G. A. 2006, ApJ, 640, 407

Li, W., Leaman, J., Chornock, R., et al. 2011, MNRAS, 412, 1441

Lira, P., Suntzeff, N. B., Phillips, M. M., et al. 1998, AJ, 115, 234

Liu, W., Jeffery, D. J., \& Schultz, D. R. 1997, ApJ, 486, L35

Malone, C. M., Nonaka, A., Woosley, S. E., et al. 2014, ApJ, 782, 11

Marquardt, K. S., Sim, S. A., Ruiter, A. J., et al. 2015, A\&A, 580, A118

Mazzali, P. A., Danziger, I. J., \& Turatto, M. 1995, A\&A, 297, 509

Meakin, C. A., Seitenzahl, I., Townsley, D., et al. 2009, ApJ, 693, 1188

Mennekens, N., Vanbeveren, D., De Greve, J. P., \& De Donder, E. 2010, A\&A, $515, \mathrm{~A} 89$

Niemeyer, J. C., \& Hillebrandt, W. 1997, in Thermonuclear Supernovae, eds.

P. Ruiz-Lapuente, R. Canal, \& J. Isern (Dordrecht: Kluwer Academic Publishers), NATO ASIC Proc., 486, 441

Nomoto, K. 1982, ApJ, 253, 798

Nonaka, A., Aspden, A. J., Zingale, M., et al. 2012, ApJ, 745, 73

Osher, S., \& Sethian, J. A. 1988, J. Comput. Phys., 79, 12 
Pakmor, R., Hachinger, S., Röpke, F. K., \& Hillebrandt, W. 2011, A\&A, 528, A117

Pakmor, R., Edelmann, P., Röpke, F. K., \& Hillebrandt, W. 2012a, MNRAS, 424, 2222

Pakmor, R., Kromer, M., Taubenberger, S., et al. 2012b, ApJ, 747, L10

Pastorello, A., Mazzali, P. A., Pignata, G., et al. 2007a, MNRAS, 377, 1531

Pastorello, A., Taubenberger, S., Elias-Rosa, N., et al. 2007b, MNRAS, 376, 1301

Phillips, M. M., Wells, L. A., Suntzeff, N. B., et al. 1992, AJ, 103, 1632

Plewa, T. 2007, ApJ, 657, 942

Plewa, T., Calder, A. C., \& Lamb, D. Q. 2004, ApJ, 612, L37

Pocheau, A. 1994, Phys. Rev. E, 49, 1109

Reinecke, M., Hillebrandt, W., Niemeyer, J. C., Klein, R., \& Gröbl, A. 1999, A\&A, 347, 724

Rest, A., Matheson, T., Blondin, S., et al. 2008, ApJ, 680, 1137

Röpke, F. K. 2005, A\&A, 432, 969

Röpke, F. K., Hillebrandt, W., Niemeyer, J. C., \& Woosley, S. E. 2006, A\&A, 448,1

Röpke, F. K., Woosley, S. E., \& Hillebrandt, W. 2007, ApJ, 660, 1344

Ruiter, A. J., Belczynski, K., \& Fryer, C. 2009, ApJ, 699, 2026

Ruiter, A. J., Belczynski, K., Sim, S. A., et al. 2011, MNRAS, 1282

Ruiter, A. J., Sim, S. A., Pakmor, R., et al. 2013, MNRAS, 429, 1425

Ruiz-Lapuente, P., Cappellaro, E., Turatto, M., et al. 1992, ApJ, 387, L33

Saha, A., Thim, F., Tammann, G. A., Reindl, B., \& Sandage, A. 2006, ApJS 165,108

Sasdelli, M., Mazzali, P. A., Pian, E., et al. 2014, MNRAS, 445, 711

Scalzo, R. A., Ruiter, A. J., \& Sim, S. A. 2014, MNRAS, 445, 2535

Schaefer, B. E., \& Pagnotta, A. 2012, Nature, 481, 164

Schmidt, B. P., Kirshner, R. P., Leibundgut, B., et al. 1994, ApJ, 434, L19

Schmidt, W., Niemeyer, J. C., \& Hillebrandt, W. 2006a, A\&A, 450, 265

Schmidt, W., Niemeyer, J. C., Hillebrandt, W., \& Röpke, F. K. 2006b, A\&A, 450,283
Seitenzahl, I. R., Meakin, C. A., Lamb, D. Q., \& Truran, J. W. 2009a, ApJ, 700, 642

Seitenzahl, I. R., Meakin, C. A., Townsley, D. M., Lamb, D. Q., \& Truran, J. W. 2009b, ApJ, 696, 515

Seitenzahl, I. R., Röpke, F. K., Fink, M., \& Pakmor, R. 2010, MNRAS, 407, 2297

Seitenzahl, I. R., Ciaraldi-Schoolmann, F., \& Röpke, F. K. 2011, MNRAS, 414 2709

Seitenzahl, I. R., Cescutti, G., Röpke, F. K., Ruiter, A. J., \& Pakmor, R. 2013a, A\&A, 559, L5

Seitenzahl, I. R., Ciaraldi-Schoolmann, F., Röpke, F. K., et al. 2013b, MNRAS, 429, 1156

Silverman, J. M., Foley, R. J., Filippenko, A. V., et al. 2012, MNRAS, 425, 1789 Sim, S. A. 2007, MNRAS, 375, 154

Sim, S. A., Seitenzahl, I. R., Kromer, M., et al. 2013, MNRAS, 436, 333

Stehle, M., Mazzali, P. A., Benetti, S., \& Hillebrandt, W. 2005, MNRAS, 360, 1231

Stephan, M., \& Docter, J. 2015, J. Large-Scale Research Facilities,

Thielemann, F.-K., Nomoto, K., \& Yokoi, K. 1986, A\&A, 158, 17

Timmes, F. X., \& Woosley, S. E. 1992, ApJ, 396, 649

Toonen, S., Nelemans, G., \& Portegies Zwart, S. 2012, A\&A, 546, A70

Townsley, D. M., Calder, A. C., Asida, S. M., et al. 2007, ApJ, 668, 1118

Travaglio, C., Hillebrandt, W., Reinecke, M., \& Thielemann, F.-K. 2004, A\&A, 425,1029

Tutukov, A., \& Yungelson, L. 1996, MNRAS, 280, 1035

Webbink, R. F. 1984, ApJ, 277, 355

Whelan, J., \& Iben, I. J. 1973, ApJ, 186, 1007

Woosley, S. E., \& Kasen, D. 2011, ApJ, 734, 38

Woosley, S. E., Wunsch, S., \& Kuhlen, M. 2004, ApJ, 607, 921

Yungelson, L. R., \& Livio, M. 2000, ApJ, 528, 108

Yungelson, L., Livio, M., Tutukov, A., \& Kenyon, S. J. 1995, ApJ, 447, 656 